International Journal of Modern Physics B

Vol. 33, No. 14 (2019) 1950147 (16 pages)

(C) The Author(s)

DOI: $10.1142 / \mathrm{S} 0217979219501479$

\title{
Infinite number of conservation laws and Darboux transformations for a 6-field integrable lattice system
}

\author{
Fangcheng Fan \\ School of Mathematics, Jilin University, \\ Changchun 130012, P. R. China \\ fanfc16@mails.jlu.edu.cn \\ Shaoyun Shi \\ School of Mathematics, Jilin University, \\ Changchun 130012, P. R. China \\ State Key Laboratory of Automotive Simulation and Control, \\ Jilin University, Changchun 130012, P. R. China \\ shisy@jlu.edu.cn \\ Zhiguo $\mathrm{Xu}^{*}$ \\ School of Mathematics, Jilin University, \\ Changchun 130012, P. R. China \\ xuzg2014@jlu.edu.cn
}

Received 4 January 2019

Revised 30 March 2019

Accepted 2 April 2019

Published 18 April 2019

\begin{abstract}
In this paper, we study a 6-field integrable lattice system, which, in some special cases, can be reduced to the self-dual network equation, the discrete second-order nonlinear Schrödinger equation and the relativistic Volterra lattice equation. With the help of the Lax pair, we construct infinitely many conservation laws and a new Darboux transformation for system. Exact solutions resulting from the obtained Darboux transformation are presented by using a given seed solution. Further, we generate the soliton solutions and plot the figures of one-soliton solutions with properly parameters.
\end{abstract}

Keywords: A 6-field integrable lattice system; conservation law; Darboux transformation; exact solution.

PACS numbers: 05.49.Yu, 02.30.1k, 02.70.Wz

This is an Open Access article published by World Scientific Publishing Company. It is distributed under the terms of the Creative Commons Attribution 4.0 (CC-BY) License. Further distribution of this work is permitted, provided the original work is properly cited.

* Corresponding author. 


\section{Introduction}

Dating back to the work of Fermi et al. in the $1950 \mathrm{~s},{ }^{1}$ nonlinear integrable lattice equations have been the focus of many nonlinear studies. Particularly, many physically interesting phenomena can be modeled with nonlinear integrable lattice equations, including particle vibrations in lattices, currents in electrical networks, pulses in biological chains, etc. The study of integrable systems of lattice versions, therefore, has aroused increasing interest in recent years. A number of physically important nonlinear integrable lattice equations have been obtained and systematically discussed, for instance the Ablowitz-Ladik lattice,,$\underset{2}{2}$ the Belov-Chaltikian lattice,,$\frac{3}{-}$ the Blaszak-Marciniak lattice,,$\stackrel{4}{ }$ and so on. There have been some studies on higher-order symmetries, Hamiltonian structures and Casoratian solutions for lattice system. ${ }^{5,6}$ The Volterra lattice and the modified Volterra lattice equation have recently been solved by Darboux transformation. ${ }^{7,8}$ Also, some active researches are interested in the kind of exact solutions to integrable equations called lumps. $.^{9}-11$

In order to model the nonlinear Frenkel-like excitations on a triangular lattice ribbon, Vakhnenko introduced the following 6 -field integrable lattice system ${ }^{12}$ :

$$
\left\{\begin{array}{l}
p_{n, t}=p_{n} q_{n} x_{n-1}-p_{n} q_{n+1} x_{n}-r_{n} x_{n-1}+r_{n} x_{n}-q_{n} y_{n}+q_{n+1} y_{n} \\
q_{n, t}=q_{n} r_{n-1} y_{n}-q_{n} q_{n+1} x_{n}+q_{n+1} z_{n}-p_{n} q_{n}-r_{n-1}+r_{n} \\
r_{n, t}=r_{n} r_{n-1} y_{n}-q_{n+1} r_{n} x_{n}-p_{n} r_{n-1}+r_{n} z_{n}-q_{n}+q_{n+1}, \\
x_{n, t}=q_{n} x_{n} x_{n-1}-r_{n} x_{n} y_{n+1}-x_{n-1} z_{n}+p_{n} x_{n}-y_{n}+y_{n+1}, \\
y_{n, t}=q_{n} x_{n-1} y_{n}-r_{n} y_{n} y_{n+1}+p_{n} y_{n+1}-y_{n} z_{n}-x_{n-1}+x_{n} \\
z_{n, t}=r_{n-1} y_{n} z_{n}-r_{n} y_{n+1} z_{n}-r_{n-1} x_{n}+r_{n} x_{n}-q_{n} y_{n}+q_{n} y_{n+1}
\end{array}\right.
$$

where $t \in \mathbb{R}$ is a continuous variable, $n \in \mathbb{N}$ is a discrete variable. Moreover, in the framework of generalized recursive approach, the author constructed the most significant local conservation laws explicitly, and presented the Hamiltonian formulation and Poisson structure for (1). He also derived the Darboux transformation by using the Darboux-dressing method, from which the multicomponent one-soliton solution was generated. In fact, by taking an appropriate choice of discrete variables, Vakhnenko found that (1) can reduce to an integrable nonlinear Schrödinger system on a triangular lattice ribbon, $\frac{13}{13}$ which had been investigated in Ref. 14 . In addition, (1) also permits at least two variants of purely real-valued reductions that yield the systems of semidiscrete KdV type understood in a sense used by Tsuchida, Ujino and Wadati. $\frac{15}{}$

Interestingly, we should point out that (1) can reduce to some famous equations in some special cases. For example, when

$$
\begin{aligned}
& p_{n}=I_{n} V_{n}, \quad q_{n}= \pm I_{n}, \quad r_{n}=V_{n}, \\
& x_{n}= \pm V_{n}, \quad y_{n}=I_{n}, \quad z_{n}=I_{n} V_{n}
\end{aligned}
$$


(1) comes to the following nonlinear self-dual network equation ${ }^{2,16}$ :

$$
\left\{\begin{array}{l}
I_{n, t}=\left(1 \pm I_{n}^{2}\right)\left( \pm V_{n-1} \mp V_{n}\right), \\
V_{n, t}=\left(1 \pm V_{n}^{2}\right)\left( \pm I_{n} \mp I_{n+1}\right),
\end{array}\right.
$$

which describes propagation of electrical signals in electric circuit. Rich mathematical structures related to (2) have been obtained. For example, the exact solution was found by inverse scattering in Ref. 2, the kink-shaped solitary solution was presented by ADM-padé technique in Ref. 17, and the $N$-fold Darboux transformation, infinite conservation laws were constructed in Ref. 18 . If we set

$$
p_{n}=R_{n} S_{n}, \quad q_{n}=\mp R_{n}^{*}, \quad r_{n}=S_{n}, \quad x_{n}=\mp S_{n}^{*}, \quad y_{n}=R_{n}, \quad z_{n}=R_{n}^{*} S_{n}^{*},
$$

and $\bar{t}=-\mathrm{i}$, the system (1) is regarded as two coupled discrete nonlinear Schrödinger equations

$$
\left\{\begin{array}{l}
R_{n, \bar{t}}=\mathrm{i}\left(1 \pm R_{n} R_{n}^{*}\right)\left(\mp S_{n}^{*} \pm S_{n-1}^{*}\right), \\
S_{n, \bar{t}}=\mathrm{i}\left(1 \pm S_{n} S_{n}^{*}\right)\left(\mp R_{n+1}^{*} \pm R_{n}^{*}\right),
\end{array}\right.
$$

which is a special reduction of the four-field Ablowitz-Ladik equation, $\frac{2}{}$ and infinite conservation laws, $N$-fold Darboux transformation, $N$-soliton solutions of (3) were obtained in Ref. 19. Let

$$
\begin{aligned}
& p_{n}=2 w_{n+1}+2 v_{n}+1, \quad q_{n}=-\left(2 v_{n}+1\right), \quad r_{n}=-\left(2 w_{n+1}+1\right), \\
& x_{n}=-\left(2 w_{n+1}+1\right), \quad y_{n}=-\left(2 v_{n}+1\right), \quad z_{n}=2 w_{n+1}+2 v_{n}+1,
\end{aligned}
$$

the system (1) becomes the relativistic Volterra lattice ${ }^{20}$

$$
\left\{\begin{array}{l}
u_{n, t}=u_{n}\left(v_{n}-v_{n-1}+u_{n} v_{n}-u_{n-1} v_{n-1}\right), \\
v_{n, t}=v_{n}\left(u_{n+1}-u_{n}+u_{n+1} v_{n+1}-u_{n} v_{n}\right) .
\end{array}\right.
$$

Mathematical structures related to (4) such as Lax pairs, $\stackrel{21}{ }$ Hamiltonian structure, master symmetries, infinitely many conservation laws, ${ }^{22}$ bilinear structure,, 23 and Darboux transformation ${ }^{24}$ have been closely studied.

In this paper, we firstly establish infinitely many conservation laws formulaically for (1) by using the Lax pair. Secondly, we derive a new Darboux transformation via the method proposed and developed by $\mathrm{Wu}$ et al. $\stackrel{25-27}{2}$ Finally, by using the obtained Darboux transformation, we generate the exact solutions of the system. Meanwhile, the soliton solutions, and the figures of one-soliton solutions with properly parameters are presented.

The rest of the paper is organized as follows. In Sec. 2, by means of the Lax pair of (1), we derive infinitely many conservation laws. In Sec. 3, the Darboux transformation of (1) is constructed by means of the gauge transformation and Lax pair. In Sec. 4, we get the exact solutions through the obtained Darboux transformation, from which we generate the soliton solutions and plot the figures of the one-soliton solutions with properly parameters. Some remarks and summary will be presented in the last section. 


\section{Lax Pair and Infinitely Many Conservation Laws}

The Lax pair corresponding to (1) can be given by the discrete spectral problem ${ }^{12}$

$$
E \phi_{n}=U_{n} \phi_{n}, \quad U_{n}=\left(\begin{array}{cc}
\lambda^{2}+p_{n} & \lambda q_{n}+\frac{1}{\lambda} r_{n} \\
\lambda x_{n}+\frac{1}{\lambda} y_{n} & z_{n}+\frac{1}{\lambda^{2}}
\end{array}\right)
$$

and the auxiliary evolution problem

$$
\phi_{n, t}=V_{n} \phi_{n}, \quad V_{n}=\left(\begin{array}{cc}
\lambda^{2}-q_{n} x_{n-1} & \lambda q_{n}+\frac{1}{\lambda} r_{n-1} \\
\lambda x_{n-1}+\frac{1}{\lambda} y_{n} & \frac{1}{\lambda^{2}}-r_{n-1} y_{n}
\end{array}\right),
$$

where the shift operator $E$ is defined by $E f(n, t)=f(n+1, t)=f_{n+1}$, $\phi_{n}=\left(\phi_{1, n}, \phi_{2, n}\right)^{\mathrm{T}}$ is eigenfunction, $\lambda$ is the spectral parameter and $\lambda_{t}=0$, $p_{n}, q_{n}, r_{n}, x_{n}, y_{n}, z_{n}$ are potential functions. The compatibility condition of (5) and (6) leads to the discrete zero curvature equation ${ }^{28}$

$$
U_{n, t}=\left(E V_{n}\right) U_{n}-U_{n} V_{n},
$$

from which we arrive at (1). Based on these facts, we know that (1) is Lax integrable.

The existence of infinite number of conservation laws is the key integrability characteristic for an integrable equation. A systematic method for finding an infinite hierarchy of conservation laws for semi-discrete nonlinear integrable systems has a long history. In Refs. 15 and 29, by using the recursive approach, infinitely many conservation laws for the coupled $\mathrm{KdV}$ equations and coupled nonlinear Schrödinger equations were derived. Motivating by the above work, Zhu et al. gave a simple approach to derive conservation laws for some semi-discrete systems from Lax pair directly. ${ }^{22,30}$ Recently, Vakhnenko modified procedure of the recursive approach, ${ }^{31,32}$ which is applicable for any semi-discrete integrable system associated with the auxiliary square spectral and evolution matrices of an arbitrary order.

In fact, the hierarchy of conservation laws for (1) had been studied by Vakhnenko in Ref. 12. Next, we will derive infinite number of conservation laws for (1) by using the method in Refs. 22 and 30, and give conservation laws formulaically.

Firstly, let $\Gamma_{n}=\frac{\phi_{2, n}}{\phi_{1, n}}$, then a direct calculation from (5) leads to the following Riccati equation

$$
\begin{gathered}
\lambda^{4} \Gamma_{n+1}+\lambda^{3} q_{n} \Gamma_{n} \Gamma_{n+1}+\lambda^{2}\left(p_{n} \Gamma_{n+1}-z_{n} \Gamma_{n}\right) \\
+\lambda r_{n} \Gamma_{n} \Gamma_{n+1}-\Gamma_{n}-\lambda^{3} x_{n}-\lambda y_{n}=0 .
\end{gathered}
$$

Suppose that

$$
\Gamma_{n}=\sum_{j=1}^{\infty} \lambda^{-j} f_{n}^{(j)}
$$


is a solution of $(7)$, where $f_{n}^{(j)}$ are the functions to be determined. Substituting (8) into (7), and comparing the coefficients of the same power of $\lambda$, we obtain

$$
\begin{aligned}
f_{n}^{(1)}= & x_{n-1}, \\
f_{n}^{(3)}= & -q_{n-1} x_{n-2} x_{n-1}-p_{n-1} x_{n-1}+x_{n-2} z_{n-1}+y_{n-1}, \\
f_{n}^{(2 j+1)}= & -q_{n-1}\left(f_{n-1}^{(1)} f_{n}^{(2 j-1)}+f_{n-1}^{(3)} f_{n}^{(2 j-3)}+\cdots+f_{n-1}^{(2 j-1)} f_{n}^{(1)}\right) \\
& +r_{n-1}\left(f_{n-1}^{(1)} f_{n}^{(2 j-3)}+f_{n-1}^{(3)} f_{n}^{(2 j-5)}+\cdots+f_{n-1}^{(2 j-3)} f_{n}^{(1)}\right) \\
& -p_{n-1} f_{n}^{(2 j-1)}+z_{n-1} f_{n-1}^{(2 j-1)}+f_{n-1}^{(2 j-3)}, \quad j=2,3 \ldots, \\
f_{n}^{(2 k)}= & 0, \quad k=1,2, \ldots
\end{aligned}
$$

A direct computation from (5) and (6) leads to

$$
\begin{aligned}
& \frac{\phi_{1, n+1}}{\phi_{1, n}}=\lambda^{2}+p_{n}+\left(\lambda q_{n}+\frac{1}{\lambda} r_{n}\right) \Gamma_{n}, \\
& \frac{\phi_{1, n, t}}{\phi_{1, n}}=\lambda^{2}-q_{n} x_{n-1}+\left(\lambda q_{n}+\frac{1}{\lambda} r_{n-1}\right) \Gamma_{n} .
\end{aligned}
$$

Note that

$$
\left(\ln \frac{\phi_{1, n+1}}{\phi_{1, n}}\right)_{t}=(E-1) \frac{\phi_{1, n, t}}{\phi_{1, n}}
$$

we get

$$
\left[\ln \left(1+\frac{1}{\lambda^{2}} \Psi_{n}\right)\right]_{t}=(E-1) \Phi_{n}
$$

where

$$
\begin{aligned}
& \Psi_{n}=p_{n}+\lambda q_{n} \Gamma_{n}+\frac{1}{\lambda} r_{n} \Gamma_{n} \triangleq \sum_{j=0}^{\infty} v_{j} \lambda^{-2 j} \\
& \Phi_{n}=-q_{n} x_{n-1}+\lambda q_{n} \Gamma_{n}+\frac{1}{\lambda} r_{n-1} \Gamma_{n} \triangleq \sum_{k=1}^{\infty} w_{k} \lambda^{-2 k}
\end{aligned}
$$

with

$$
\begin{aligned}
& v_{0}=p_{n}+q_{n} f_{n}^{(1)}, \quad v_{j}=r_{n} f_{n}^{(2 j-1)}+q_{n} f_{n}^{(2 j+1)}, \quad j=0,1, \ldots, \\
& w_{1}=-q_{n} x_{n-1}+q_{n} f_{n}^{(1)}, \quad w_{k}=r_{n-1} f_{n}^{(2 j-1)}+q_{n} f_{n}^{(2 j+1)}, \quad k=1,2, \ldots
\end{aligned}
$$

Now substituting $\Psi_{n}$ and $\Phi_{n}$ into (9), and making a comparison of the same powers of $\lambda$ on both sides, we obtain the following infinitely many conservation laws for (1):

$$
\rho_{n, t}^{(j)}=(E-1) J_{n}^{(j)}
$$


where

$$
\begin{aligned}
& \rho_{n}^{(1)}=v_{0}, \quad J_{n}^{(1)}=w_{1} \\
& \rho_{n}^{(2)}=v_{1}-\frac{1}{2} v_{0}^{2}, \quad J_{n}^{(2)}=w_{2} \\
& \rho_{n}^{(j)}=v_{j-1}-\frac{1}{2} \sum_{l_{1}+l_{2}=j-2} v_{l_{1}} v_{l_{2}}+\cdots+(-1)^{j-2} v_{0}^{j-2} v_{1}+\frac{(-1)^{j-1}}{j} v_{0}^{j}, \\
& J_{n}^{(j)}=w_{j}, \quad j=3,4, \ldots
\end{aligned}
$$

Secondly, let $\tilde{\Gamma}_{n}=\frac{\phi_{1, n}}{\phi_{2, n}}$, then a direct calculation from (5) leads to the following Riccati equation:

$$
\begin{aligned}
& \tilde{\Gamma}_{n+1}+\lambda y_{n} \tilde{\Gamma}_{n} \tilde{\Gamma}_{n+1}+\lambda^{2}\left(z_{n} \tilde{\Gamma}_{n+1}-p_{n} \tilde{\Gamma}_{n}\right) \\
& \quad+\lambda^{3} x_{n} \tilde{\Gamma}_{n} \tilde{\Gamma}_{n+1}+\lambda^{4} \tilde{\Gamma}_{n}-\lambda^{3} q_{n}-\lambda r_{n}=0 .
\end{aligned}
$$

Suppose that

$$
\tilde{\Gamma}_{n}=\sum_{j=1}^{\infty} \lambda^{j} \tilde{f}_{n}^{(j)}
$$

is a solution of (11), where $\tilde{f}_{n}^{(j)}$ are the functions to be determined. Substituting (12) into (11), and making a comparison of the powers of $\lambda$ on both sides of equation (11), we obtain

$$
\begin{aligned}
\tilde{f}_{n}^{(1)}= & r_{n-1}, \\
\tilde{f}_{n}^{(3)}= & -y_{n-1} r_{n-2} r_{n-1}-z_{n-1} r_{n-1}+r_{n-2} p_{n-1}+q_{n-1}, \\
\tilde{f}_{n}^{(2 j+1)}= & -y_{n-1}\left(\tilde{f}_{n-1}^{(1)} \tilde{f}_{n}^{(2 j-1)}+\tilde{f}_{n-1}^{(3)} \tilde{f}_{n}^{(2 j-3)}+\cdots+\tilde{f}_{n-1}^{(2 j-1)} \tilde{f}_{n}^{(1)}\right) \\
& -x_{n-1}\left(\tilde{f}_{n-1}^{(1)} \tilde{f}_{n}^{(2 j-3)}+\tilde{f}_{n-1}^{(3)} \tilde{f}_{n}^{(2 j-5)}+\cdots+\tilde{f}_{n-1}^{(2 j-3)} \tilde{f}_{n}^{(1)}\right) \\
& -z_{n-1} \tilde{f}_{n}^{(2 j-1)}+p_{n-1} \tilde{f}_{n-1}^{(2 j-1)}-\tilde{f}_{n-1}^{(2 j-3)}, \quad j=2,3 \ldots, \\
\tilde{f}_{n}^{(2 k)}= & 0, \quad k=1,2, \ldots
\end{aligned}
$$

In this case, we obtain the following infinitely many conservation laws for (1):

$$
\tilde{\rho}_{n, t}^{(j)}=(E-1) \tilde{J}_{n}^{(j)}
$$

where

$$
\begin{aligned}
& \tilde{\rho}_{n}^{(1)}=\tilde{v}_{0}, \quad \tilde{J}_{n}^{(1)}=\tilde{w}_{1} \\
& \tilde{\rho}_{n}^{(2)}=\tilde{v}_{1}-\frac{1}{2} \tilde{v}_{0}^{2}, \quad \tilde{J}_{n}^{(2)}=\tilde{w}_{2} \\
& \tilde{\rho}_{n}^{(j)}=\tilde{v}_{j-1}-\frac{1}{2} \sum_{l_{1}+l_{2}=j-2} \tilde{v}_{l_{1}} \tilde{v}_{l_{2}}+\cdots+(-1)^{j-2} \tilde{v}_{0}^{j-2} \tilde{v}_{1}+\frac{(-1)^{j-1}}{j} \tilde{v}_{0}^{j} \\
& \tilde{J}_{n}^{(j)}=\tilde{w}_{j}, \quad j=3,4, \ldots
\end{aligned}
$$


with

$$
\begin{aligned}
& \tilde{v}_{0}=z_{n}+y_{n} \tilde{f}_{n}^{(1)}, \quad \tilde{v}_{j}=x_{n} \tilde{f}_{n}^{(2 j-1)}+y_{n} \tilde{f}_{n}^{(2 j+1)}, \quad j=0,1, \ldots, \\
& \tilde{w}_{1}=x_{n-1} \tilde{f}_{n}^{(1)}+y_{n} \tilde{f}_{n}^{(3)}, \quad \tilde{w}_{k}=x_{n-1} \tilde{f}_{n}^{(2 k-1)}+y_{n} \tilde{f}_{n}^{(2 k+1)}, \quad k=1,2, \ldots
\end{aligned}
$$

Remark 2.1. We point out that referring onto the already known general formulas for the collection of generating equations and the set of Riccati equation presented in Ref. 12, the hierarchy of infinite number of conservation laws for system (1) can also be obtained.

Remark 2.2. The infinite number of conservation laws (10) and (13) for system (1) satisfy the symmetry between the field variables $p_{n} \leftrightarrows \overline{z_{n}}, q_{n} \leftrightarrows \overline{y_{n}}, r_{n} \leftrightarrows x_{n}$

\section{Darboux Transformation}

In this section, we proceed to establish the Darboux transformation for (1). In fact, Darboux transformation is a special gauge transformation of the spectral problem and transforms the spectral problem into another spectral problem of the same type. $\frac{33-35}{-}$ For this, we introduce following gauge transformation:

$$
\tilde{\phi}_{n}=T_{n} \phi_{n}
$$

which can transform the spectral problems (5) and (6) into

$$
E \tilde{\phi}_{n}=\tilde{U}_{n} \tilde{\varphi}_{n}, \quad \tilde{\phi}_{n, t}=\tilde{V}_{n} \tilde{\varphi}_{n}
$$

where

$$
\tilde{U}_{n}=T_{n+1} U_{n} T_{n}^{-1}, \quad \tilde{V}_{n}=\left(T_{n t}+T_{n} V_{n}\right) T_{n}^{-1},
$$

and the matrixes $\tilde{U}_{n}, \tilde{V}_{n}$ have the same structures as $U_{n}$ and $V_{n}$, respectively. We shall construct Darboux transformation as follows.

Assume that $\left(p_{n}, q_{n}, r_{n}, x_{n}, y_{n}, z_{n}\right)$ is a given solution to $(1)$, and $\varphi_{n}(\lambda)=$ $\left(\varphi_{1, n}(\lambda), \varphi_{2, n}(\lambda)\right)^{\mathrm{T}}, \psi_{n}(\lambda)=\left(\psi_{1, n}(\lambda), \psi_{2, n}(\lambda)\right)^{\mathrm{T}}$ are two linear independent solutions of (5) and (6). Define

$$
\sigma_{i, n}=\frac{\varphi_{2, n}\left(\lambda_{i}\right)+\gamma_{i} \psi_{2, n}\left(\lambda_{i}\right)}{\varphi_{1, n}\left(\lambda_{i}\right)+\gamma_{i} \psi_{1, n}\left(\lambda_{i}\right)}, \quad i=1,2
$$

and

$$
\begin{aligned}
& a_{n}=\frac{\lambda_{1} \lambda_{2}\left(\lambda_{1} \sigma_{2, n}-\lambda_{2} \sigma_{1, n}\right)}{\lambda_{1} \sigma_{1, n}-\lambda_{2} \sigma_{2, n}}, \quad b_{n}=\frac{\lambda_{2}^{2}-\lambda_{1}^{2}}{\lambda_{1} \sigma_{1, n}-\lambda_{2} \sigma_{2, n}}, \\
& c_{n}=\frac{\sigma_{1, n} \sigma_{2, n}\left(\lambda_{2}^{2}-\lambda_{1}^{2}\right)}{\lambda_{1} \lambda_{2}\left(\lambda_{1} \sigma_{1, n}-\lambda_{2} \sigma_{2, n}\right)}, \quad d_{n}=\frac{\lambda_{1} \sigma_{2, n}-\lambda_{2} \sigma_{1, n}}{\lambda_{1} \lambda_{2}\left(\lambda_{1} \sigma_{1, n}-\lambda_{2} \sigma_{2, n}\right)},
\end{aligned}
$$

where parameters $\lambda_{i}$ and $\gamma_{i}\left(\lambda_{1} \neq \lambda_{2} \neq 0, \gamma_{1} \neq \gamma_{2}\right)$ are suitably chosen such that all the denominators in (14) and (15) are not zero. From (5) and (14), we have

$$
\sigma_{i, n+1}=\frac{\mu_{i, n}}{\nu_{i, n}}, \quad i=1,2,
$$


where

$$
\mu_{i, n}=\lambda_{i} x_{n}+\frac{y_{n}}{\lambda_{i}}+\left(z_{n}+\frac{1}{\lambda_{i}^{2}}\right) \sigma_{i, n}, \quad \nu_{i, n}=\lambda_{i}^{2}+p_{n}+\left(\lambda_{i} q_{n}+\frac{r_{n}}{\lambda_{i}}\right) \sigma_{i, n} .
$$

Shifting (15) by operator $E$, we get

$$
\begin{array}{ll}
a_{n+1}=\frac{\lambda_{1} \lambda_{2}\left(\lambda_{1} \sigma_{2, n+1}-\lambda_{2} \sigma_{1, n+1}\right)}{\lambda_{1} \sigma_{1, n+1}-\lambda_{2} \sigma_{2, n+1}}, & b_{n+1}=\frac{\lambda_{2}^{2}-\lambda_{1}^{2}}{\lambda_{1} \sigma_{1, n+1}-\lambda_{2} \sigma_{2, n+1}}, \\
c_{n+1}=\frac{\sigma_{1, n+1} \sigma_{2, n+1}\left(\lambda_{2}^{2}-\lambda_{1}^{2}\right)}{\lambda_{1} \lambda_{2}\left(\lambda_{1} \sigma_{1, n+1}-\lambda_{2} \sigma_{2, n+1}\right)}, & d_{n+1}=\frac{\lambda_{1} \sigma_{2, n+1}-\lambda_{2} \sigma_{1, n+1}}{\lambda_{1} \lambda_{2}\left(\lambda_{1} \sigma_{1, n+1}-\lambda_{2} \sigma_{2, n+1}\right)} .
\end{array}
$$

It is obvious that the matrix $T_{n}$ is a key step for constructing Darboux transformation, a proper $T_{n}$ will ensure the correctness of Darboux transformation. Making use of (15), we take $T_{n}$ as

$$
T_{n}=\left(\begin{array}{cc}
\lambda^{2}+a_{n} & \lambda b_{n} \\
\lambda c_{n} & 1+\lambda^{2} d_{n}
\end{array}\right) .
$$

Through calculations, we can verify from (15) that $\pm \lambda_{1}$ and $\pm \lambda_{2}$ are all the roots of the determinant of $T_{n}$. Therefore, we have

$$
\operatorname{det} T_{n}=d_{n}\left(\lambda+\lambda_{1}\right)\left(\lambda-\lambda_{1}\right)\left(\lambda+\lambda_{2}\right)\left(\lambda-\lambda_{2}\right) .
$$

In order to construct the Darboux transformation successfully, we need to ensure that $\tilde{U}_{n}$ and $\tilde{V}_{n}$ have the same forms as $U_{n}$ and $V_{n}$, respectively. We first show that the structure of $U_{n}$ is the same as $\tilde{U}_{n}$. Let

$$
F_{n}(\lambda)=T_{n+1} U_{n} T_{n}^{*}=\left(\begin{array}{ll}
F_{n}^{1}(\lambda) & F_{n}^{2}(\lambda) \\
F_{n}^{3}(\lambda) & F_{n}^{4}(\lambda)
\end{array}\right),
$$

where $T_{n}^{*}$ denotes the adjoint matrix of $T_{n}, F_{n}^{j}(\lambda)(j=1,2,3,4)$ are polynomials in $\lambda$, and the details of $F_{n}^{j}(\lambda)$ are omitted. From (14) and (15), we have

$$
\lambda_{i}^{2}+a_{n}+\lambda_{i} \sigma_{i, n} b_{n}=0, \quad \lambda_{i} c_{n}+\sigma_{i, n}+\lambda_{i}^{2} \sigma_{i, n} d_{n}=0, \quad i=1,2 .
$$

By using (15) and (20), it can be verified that $\pm \lambda_{1}$ and $\pm \lambda_{2}$ are the roots of $F_{n}^{j}(\lambda)$ $(j=1,2,3,4)$. Take into account the order of $F_{n}^{j}(\lambda)$ and (19), we conclude that there exists a matrix $P_{n}(\lambda)$ such that

$$
T_{n+1} U_{n} T_{n}^{*}=\operatorname{det} T_{n} \cdot P_{n}(\lambda),
$$

where

$$
P_{n}(\lambda)=\left(\begin{array}{cc}
\lambda^{2} P_{n}^{1}+P_{n}^{2} & \lambda P_{n}^{3}+\frac{P_{n}^{4}}{\lambda} \\
\lambda P_{n}^{5}+\frac{P_{n}^{6}}{\lambda} & P_{n}^{7}+\frac{P_{n}^{8}}{\lambda^{2}}
\end{array}\right),
$$


with $P_{n}^{k}(k=1,2, \ldots, 8)$ are independent of $\lambda$. To determine $P_{n}^{k}$, we rewrite $(21)$ as

$$
T_{n+1} U_{n}=P_{n} T_{n} .
$$

Equating the coefficients of $\lambda^{j}(j=4,3, \ldots,-2)$ in (22) we have

$$
\begin{aligned}
& P_{n}^{1}=1, \quad P_{n}^{2}=p_{n}+b_{n+1} x_{n}+a_{n+1}-a_{n}-\frac{c_{n}\left(\overline{q_{n}}-b_{n}\right)}{d_{n}}, \quad P_{n}^{3}=\frac{q_{n}-b_{n}}{d_{n}}, \\
& P_{n}^{4}=a_{n+1} r_{n}+b_{n+1}, \quad P_{n}^{5}=d_{n+1} x_{n}+c_{n+1}, \quad P_{n}^{6}=\frac{y_{n}-c_{n}}{a_{n}}, \\
& P_{n}^{7}=\frac{d_{n+1} z_{n}+c_{n+1} q_{n}-b_{n} c_{n+1}-b_{n} d_{n+1} x_{n}}{d_{n}}, \quad P_{n}^{8}=1 .
\end{aligned}
$$

Let

$$
\begin{aligned}
& \tilde{p}_{n}=p_{n}+b_{n+1} x_{n}+a_{n+1}-a_{n}-\frac{c_{n}\left(q_{n}-b_{n}\right)}{d_{n}}, \quad \tilde{q}_{n}=\frac{q_{n}-b_{n}}{d_{n}}, \\
& \tilde{r}_{n}=a_{n+1} r_{n}+b_{n+1}, \quad \tilde{x}_{n}=d_{n+1} x_{n}+c_{n+1}, \\
& \tilde{y}_{n}=\frac{y_{n}-c_{n}}{a_{n}}, \quad \tilde{z}_{n}=\frac{d_{n+1} z_{n}+c_{n+1} q_{n}-b_{n} c_{n+1}-b_{n} d_{n+1} x_{n}}{d_{n}},
\end{aligned}
$$

then

$$
P_{n}^{2}=\tilde{p}_{n}, \quad P_{n}^{3}=\tilde{q}_{n}, \quad P_{n}^{4}=\tilde{r}_{n}, \quad P_{n}^{5}=\tilde{x}_{n}, \quad P_{n}^{6}=\tilde{y}_{n}, \quad P_{n}^{7}=\tilde{z}_{n},
$$

which means

$$
\tilde{U}_{n}=T_{n+1} U_{n} T_{n}^{-1}=P_{n}(\lambda)=\left(\begin{array}{cc}
\lambda^{2}+\tilde{p}_{n} & \lambda \tilde{q}_{n}+\frac{1}{\lambda} \tilde{r}_{n} \\
\lambda \tilde{x}_{n}+\frac{1}{\lambda} \tilde{y}_{n} & \tilde{z}_{n}+\frac{1}{\lambda^{2}}
\end{array}\right) .
$$

Thus, we show that the matrix $\tilde{U}_{n}$ has the same form as $U_{n}$.

Proceeding in a similar manner, we can show that $\tilde{V}_{n}$ has the same structure with $V_{n}$. Let

$$
G_{n}(\lambda)=\left(T_{n, t}+T_{n} V_{n}\right) T_{n}^{*}=\left(\begin{array}{ll}
G_{n}^{1}(\lambda) & G_{n}^{2}(\lambda) \\
G_{n}^{3}(\lambda) & G_{n}^{4}(\lambda)
\end{array}\right),
$$

where $T_{n}^{*}$ denotes the adjoint matrix of $T_{n}$, and the details of $G_{n}^{j}(\lambda)(j=1,2,3,4)$ are omitted. By virtue of (6) and (14), we obtain

$$
\begin{aligned}
\sigma_{i, n, t}= & \lambda_{i} x_{n-1}+\frac{y_{n}}{\lambda_{i}}+\left(\frac{1}{\lambda_{i}^{2}}-r_{n-1} y_{n}-\lambda_{i}^{2}+q_{n} x_{n-1}\right) \sigma_{i, n} \\
& -\left(\lambda_{i} q_{n}+\frac{r_{n-1}}{\lambda_{i}}\right) \sigma_{i, n}^{2}, \quad i=1,2 .
\end{aligned}
$$

Deriving on both sides of (20), we have

$$
\begin{aligned}
& a_{n, t}=-\lambda_{i} b_{n, t} \sigma_{i, n}-\lambda_{i} b_{n} \sigma_{i, n, t} \\
& c_{n, t}=-\frac{1}{\lambda_{i}} \sigma_{i, n, t}-\lambda_{i} d_{n, t} \sigma_{i, n}-\lambda d_{n} \sigma_{i, n, t}, \quad i=1,2 .
\end{aligned}
$$


With the help of (20), (24) and (25), it can be verified that $\pm \lambda_{1}, \pm \lambda_{2}$ are the roots of $G_{n}^{j}(\lambda)$. Hence, there exists a matrix $Q_{n}(\lambda)$ such that

$$
\left(T_{n, t}+T_{n} V_{n}\right) T_{n}^{*}=\operatorname{det} T_{n} \cdot Q_{n}(\lambda)
$$

with

$$
Q_{n}(\lambda)=\left(\begin{array}{cc}
Q_{n}^{1} \lambda^{2}+Q_{n}^{2} & Q_{n}^{3} \lambda+\frac{1}{\lambda} Q_{n}^{4} \\
Q_{n}^{5} \lambda+\frac{1}{\lambda} Q_{n}^{6} & Q_{n}^{7}+\frac{1}{\lambda^{2}} Q_{n}^{8}
\end{array}\right)
$$

where $Q_{n}^{k}(k=1,2, \ldots, 8)$ are undetermined functions independent of $\lambda$. In order to obtain $Q_{n}^{k}$, we rewrite $(26)$ as

$$
T_{n, t}+T_{n} V_{n}=Q_{n} T_{n}
$$

Comparing the coefficients of $\lambda^{j}(j=4,3, \ldots,-2)$ in $(27)$, we have

$$
\begin{aligned}
& Q_{n}^{1}=1, \quad Q_{n}^{2}=b_{n} x_{n-1}-q_{n} x_{n-1}-\frac{c_{n}\left(q_{n}-b_{n}\right)}{d_{n}}, \\
& Q_{n}^{3}=\frac{q_{n}-b_{n}}{d_{n}}, \quad Q_{n}^{4}=b_{n}+a_{n} r_{n-1}, \\
& Q_{n}^{5}=c_{n}+d_{n} x_{n-1}, \quad Q_{n}^{6}=\frac{y_{n}-c_{n}}{a_{n}}, \\
& Q_{n}^{7}=c_{n} r_{n-1}-r_{n-1} y_{n}-\frac{b_{n}\left(y_{n}-c_{n}\right)}{a_{n}}, \quad Q_{n}^{8}=1 .
\end{aligned}
$$

By (23) and (28), we can verify

$$
\begin{aligned}
& Q_{n}^{2}=-\tilde{q}_{n} \tilde{x}_{n-1}, \quad Q_{n}^{3}=\tilde{q}_{n}, \quad Q_{n}^{4}=\tilde{r}_{n-1}, \\
& Q_{n}^{5}=\tilde{x}_{n-1}, \quad Q_{n}^{6}=\tilde{y}_{n}, \quad Q_{n}^{7}=-\tilde{r}_{n-1} \tilde{y}_{n},
\end{aligned}
$$

so

$$
\tilde{V}_{n}=\left(T_{n, t}+T_{n} V_{n}\right) T_{n}^{-1}=\left(\begin{array}{cc}
\lambda^{2}-\tilde{q}_{n} \tilde{x}_{n-1} & \lambda \tilde{q}_{n}+\frac{1}{\lambda} \tilde{r}_{n-1} \\
\lambda \tilde{x}_{n-1}+\frac{1}{\lambda} \tilde{y}_{n} & \frac{1}{\lambda^{2}}-\tilde{r}_{n-1} \tilde{y}_{n}
\end{array}\right) .
$$

Therefore, the matrix $\tilde{V}_{n}$ has the same form as $V_{n}$.

Based on the above facts, we can now conclude the following theorem.

Theorem 3.1. $\left(\phi_{n}, p_{n}, q_{n}, r_{n}, x_{n}, y_{n}, z_{n}\right) \rightarrow\left(\tilde{\phi}_{n}, \tilde{p}_{n}, \tilde{q}_{n}, \tilde{r}_{n}, \tilde{x}_{n}, \tilde{y}_{n}, \tilde{z}_{n}\right)$ is a Darboux transformation of (1). Furthermore, if $\left(p_{n}, q_{n}, r_{n}, x_{n}, y_{n}, z_{n}\right)$ are the solutions 
of (1), then $\left(\tilde{p}_{n}, \tilde{q}_{n}, \tilde{r}_{n}, \tilde{x}_{n}, \tilde{y}_{n}, \tilde{z}_{n}\right)$ are the new solutions under the transformation

$$
\begin{aligned}
& \tilde{p}_{n}=p_{n}+b_{n+1} x_{n}+a_{n+1}-a_{n}-\frac{c_{n}\left(q_{n}-b_{n}\right)}{d_{n}}, \\
& \tilde{q}_{n}=\frac{q_{n}-b_{n}}{d_{n}}, \quad \tilde{r}_{n}=a_{n+1} r_{n}+b_{n+1} \\
& \tilde{x}_{n}=d_{n+1} x_{n}+c_{n+1}, \quad \tilde{y}_{n}=\frac{y_{n}-c_{n}}{a_{n}} \\
& \tilde{z}_{n}=\frac{d_{n+1} z_{n}+c_{n+1} q_{n}-b_{n} c_{n+1}-b_{n} d_{n+1} x_{n}}{d_{n}}
\end{aligned}
$$

Remark 3.1. We point out that Vakhnenko also constructed the Darboux transformation of system (1) through the following Darboux matrix ${ }^{12,14}$ :

$$
S(n \mid \lambda)=\left(\begin{array}{cc}
\lambda^{2}+u_{11}(n) & t_{12}(n) \lambda+u_{12}(n) \lambda^{-1} \\
t_{21}(n) \lambda+u_{21}(n) \lambda^{-1} & t_{22}(n)+\lambda^{-2}
\end{array}\right),
$$

which satisfies the interchange $\lambda \rightleftharpoons \frac{1}{\lambda}$ of spectral parameters and symmetries between the field variables $p_{n} \rightleftharpoons z_{n}, q_{n} \rightleftharpoons y_{n}, r_{n} \rightleftharpoons x_{n}$. In Sec. 3, we establish explicit Darboux transformation of system (1) by using of the Darboux matrix (18) with four Darboux functions. Considering this interchange and symmetries, the Darboux matrix we consider is not unique and there exist alternative form with the preferable use of parameter $\frac{1}{\lambda}$.

\section{Exact Solutions}

The aim of this section is to derive some explicit exact solutions for (1) by applying the Darboux transformation obtained in Sec. 3. Obviously, (1) has a seed solution $p_{n}=1, q_{n}=0, r_{n}=1, x_{n}=0, y_{n}=1, z_{n}=0$, and the Lax pair related to the seed solution is

$$
E \phi_{n}=\left(\begin{array}{cc}
\lambda^{2}+1 & \frac{1}{\lambda} \\
\frac{1}{\lambda} & \frac{1}{\lambda^{2}}
\end{array}\right) \phi_{n}, \quad \phi_{n, t}=\left(\begin{array}{cc}
\lambda^{2} & \frac{1}{\lambda} \\
\frac{1}{\lambda} & \frac{1}{\lambda^{2}}-1
\end{array}\right) \phi_{n}
$$

We can obtain two linearly independent solutions of (30) as

$$
\begin{aligned}
& \left(\begin{array}{l}
\varphi_{1, n}(\lambda) \\
\varphi_{2, n}(\lambda)
\end{array}\right)=\alpha^{n} e^{\tau t}\left(\begin{array}{c}
1 \\
-\lambda^{3}-\lambda+\lambda \alpha
\end{array}\right), \\
& \left(\begin{array}{l}
\psi_{1, n}(\lambda) \\
\psi_{2, n}(\lambda)
\end{array}\right)=\beta^{n} e^{\rho t}\left(\begin{array}{c}
1 \\
-\lambda^{3}-\lambda+\lambda \beta
\end{array}\right),
\end{aligned}
$$


where

$$
\begin{aligned}
& \alpha=\alpha(\lambda)=\frac{\lambda^{2}+\frac{1}{\lambda^{2}}+1+\sqrt{\lambda^{4}+\frac{1}{\lambda^{4}}+2 \lambda^{2}+\frac{2}{\lambda^{2}}-1}}{2}, \\
& \tau=\tau(\lambda)=\frac{\lambda^{2}+\frac{1}{\lambda^{2}}-1+\sqrt{\lambda^{4}+\frac{1}{\lambda^{4}}+2 \lambda^{2}+\frac{2}{\lambda^{2}}-1}}{2}, \\
& \beta=\beta(\lambda)=\frac{\lambda^{2}+\frac{1}{\lambda^{2}}+1-\sqrt{\lambda^{4}+\frac{1}{\lambda^{4}}+2 \lambda^{2}+\frac{2}{\lambda^{2}}-1}}{2}, \\
& \rho=\rho(\lambda)=\frac{\lambda^{2}+\frac{1}{\lambda^{2}}-1-\sqrt{\lambda^{4}+\frac{1}{\lambda^{4}}+2 \lambda^{2}+\frac{2}{\lambda^{2}}-1}}{2} .
\end{aligned}
$$

It follows from (14) and (16) that

$$
\begin{aligned}
\sigma_{i, n} & =\frac{\left[-\lambda_{i}^{3}-\lambda_{i}+\lambda_{i} \alpha_{i}\right] \alpha_{i}^{n} e^{\tau_{i} t}+\gamma_{i}\left[-\lambda_{i}^{3}-\lambda_{i}+\lambda_{i} \beta_{i}\right] \beta_{i}^{n} e^{\rho_{i} t}}{\alpha_{i}^{n} e^{\tau_{i} t}+\gamma_{i} \beta_{i}^{n} e^{\rho_{i} t}}, \\
\mu_{i, n} & =\frac{1}{\lambda_{i}}+\frac{1}{\lambda_{i}^{2}} \sigma_{i, n}, \quad \nu_{i, n}=\lambda_{i}^{2}+1+\frac{1}{\lambda_{i}} \sigma_{i, n},
\end{aligned}
$$

where

$$
\alpha_{i}=\alpha\left(\lambda_{i}\right), \quad \beta_{i}=\beta\left(\lambda_{i}\right), \quad \tau_{i}=\tau\left(\lambda_{i}\right), \quad \rho_{i}=\rho\left(\lambda_{i}\right), \quad i=1,2 .
$$

By using Theorem 3.1, the explicit exact solutions of system (1) are given by

$$
\left\{\begin{array}{c}
\left(\lambda_{1} \lambda_{2}\left(\lambda_{1} \sigma_{1, n}-\lambda_{2} \sigma_{2, n}\right)+\sigma_{1, n} \sigma_{2, n}\left(\lambda_{1}^{2}-\lambda_{2}^{2}\right)\right) \\
\tilde{p}_{n}=-\frac{\left(\lambda_{1}^{3} \lambda_{2}-\lambda_{1} \lambda_{2}^{3}+\lambda_{2} \sigma_{1, n}-\lambda_{1} \sigma_{2, n}\right)}{\left(\lambda_{1} \sigma_{2, n}-\lambda_{2} \sigma_{1, n}\right)\left(\lambda_{1} \lambda_{2}^{3}-\lambda_{1}^{3} \lambda_{2}+\lambda_{2}^{3} \sigma_{1, n}-\lambda_{1}^{3} \sigma_{2, n}\right)}, \\
\tilde{q}_{n}=\frac{\lambda_{1} \lambda_{2}\left(\lambda_{1}^{2}-\lambda_{2}^{2}\right)}{\lambda_{1} \sigma_{2, n}-\lambda_{2} \sigma_{1, n}}, \\
\tilde{r}_{n}=-\frac{\lambda_{1}^{2} \lambda_{2}^{2}\left(\lambda_{1}^{3} \lambda_{2}-\lambda_{1} \lambda_{2}^{3}+\lambda_{2} \sigma_{1, n}-\lambda_{1} \sigma_{2, n}\right)}{\lambda_{1} \lambda_{2}^{3}-\lambda_{1}^{3} \lambda_{2}+\lambda_{2}^{3} \sigma_{1, n}-\lambda_{1}^{3} \sigma_{2, n}}, \\
\tilde{x}_{n}=-\frac{\left(\lambda_{1}^{2}-\lambda_{2}^{2}\right)\left(\lambda_{1} \lambda_{2}+\lambda_{1} \sigma_{2, n}+\lambda_{2} \sigma_{1, n}+\sigma_{1, n} \sigma_{2, n}\right)}{\lambda_{1}^{2} \lambda_{2}^{2}\left(\lambda_{1} \lambda_{2}^{3}-\lambda_{1}^{3} \lambda_{2}+\lambda_{2}^{3} \sigma_{1, n}-\lambda_{1}^{3} \sigma_{2, n}\right)} \\
\tilde{y}_{n}=\frac{\lambda_{1} \lambda_{2}\left(\lambda_{1} \sigma_{1, n}-\lambda_{2} \sigma_{2, n}\right)+\sigma_{1, n} \sigma_{2, n}\left(\lambda_{1}^{2}-\lambda_{2}^{2}\right)}{\lambda_{1}^{2} \lambda_{2}^{2}\left(\lambda_{1} \sigma_{2, n}-\lambda_{2} \sigma_{1, n}\right)}, \\
\tilde{z}_{n}=-\frac{\left(\lambda_{1}^{2}-\lambda_{2}^{2}\right)^{2}\left(\lambda_{1} \lambda_{2}+\lambda_{1} \sigma_{2, n}+\lambda_{2} \sigma_{1, n}+\sigma_{1, n} \sigma_{2, n}\right)}{\lambda_{1} \lambda_{2}\left(\lambda_{1} \sigma_{2, n}-\lambda_{2} \sigma_{1, n}\right)\left(\lambda_{1} \lambda_{2}^{3}-\lambda_{1}^{3} \lambda_{2}+\lambda_{2}^{3} \sigma_{1, n}-\lambda_{1}^{3} \sigma_{2, n}\right)}
\end{array}\right.
$$

A direct computation from (31) leads to the following relations

$$
\tilde{p}_{n}=\tilde{r}_{n} \tilde{y}_{n}, \quad \tilde{z}_{n}=\tilde{q}_{n} \tilde{x}_{n},
$$

which means the solutions $\tilde{p}_{n}$ and $\tilde{z}_{n}$ are dependent of the solutions $\tilde{q}_{n}, \tilde{r}_{n}, \tilde{x}_{n}, \tilde{y}_{n}$. 
As we known, the soliton solutions with strictly defined free physical parameters are very meaningful and valuable in the physical applications. In what follows, we proceed to establish the soliton solutions of system (1) by means of (31). When we choose $\lambda_{2}=\frac{1}{\lambda_{1}}\left(\lambda_{1} \neq \pm i, \pm 1\right)$, we find that $\alpha_{1}=\alpha_{2}, \beta_{1}=\beta_{2}, \tau_{1}=\tau_{2}, \rho_{1}=\rho_{2}$, thus we can rewrite $\sigma_{1, n}$ and $\sigma_{2, n}$ as

$$
\begin{aligned}
\sigma_{1, n} & =\frac{\left(-\lambda_{1}^{3}-\lambda_{1}+\lambda_{1} \alpha_{1}\right)+\gamma_{1}\left(-\lambda_{1}^{3}-\lambda_{1}+\lambda_{1} \beta_{1}\right) e^{k n+w t}}{1+\gamma_{1} e^{k n+w t}} \\
\sigma_{2, n} & =\frac{\left(-\lambda_{1}^{-3}-\lambda_{1}^{-1}+\lambda_{1}^{-1} \alpha_{1}\right)+\gamma_{2}\left(-\lambda_{i}^{-3}-\lambda_{1}^{-1}+\lambda_{1}^{-1} \beta_{1}\right) e^{k n+w t}}{1+\gamma_{2} e^{k n+w t}}
\end{aligned}
$$

with $k=\ln \frac{\beta_{1}}{\alpha_{1}}, w=\rho_{1}-\tau_{1}$. From (31) and (33), we obtain

$$
\begin{aligned}
& \tilde{q}_{n}=\frac{\tilde{\Theta}_{1}+e^{-(k n+w t)}+\gamma_{1} \gamma_{2} e^{k n+w t}}{\Theta_{1}+e^{-(k n+w t)}+\gamma_{1} \gamma_{2} e^{k n+w t}}, \\
& \tilde{r}_{n}=\frac{\tilde{\Theta}_{2}}{\Theta_{2}-\alpha_{1}\left(\lambda_{1}^{2}-\lambda_{1}^{-2}\right) e^{-(k n+w t)}-\gamma_{1} \gamma_{2} \beta_{1}\left(\lambda_{1}^{2}-\lambda_{1}^{-2}\right) e^{k n+w t}}, \\
& \tilde{x}_{n}=\frac{\tilde{\Theta}_{3}+\alpha_{1} e^{-(k n+w t)}+\gamma_{1} \gamma_{2} \beta_{1} e^{k n+w t}}{\Theta_{3}+\alpha_{1} e^{-(k n+w t)}+\gamma_{1} \gamma_{2} \beta_{1} e^{k n+w t}}, \\
& \tilde{y}_{n}=\frac{\tilde{\Theta}_{4}}{\Theta_{4}+\left(\lambda_{1}^{2}-\lambda_{1}^{-2}\right) e^{-(k n+w t)}+\gamma_{1} \gamma_{2}\left(\lambda_{1}^{2}-\lambda_{1}^{-2}\right) e^{k n+w t}},
\end{aligned}
$$

where

$$
\begin{aligned}
\Theta_{1}= & \gamma_{1}+\gamma_{2}-\frac{\left(\gamma_{1}-\gamma_{2}\right)\left(\alpha_{1}-\beta_{1}\right)}{\lambda_{1}^{2}-\lambda_{1}^{-2}}, \\
\tilde{\Theta}_{1}= & \gamma_{1}+\gamma_{2}, \\
\Theta_{2}= & \gamma_{1} \beta_{1} \lambda_{1}^{-2}-\gamma_{2} \beta_{1} \lambda_{1}^{2}+\gamma_{2} \alpha_{1} \lambda_{1}^{-2}-\gamma_{1} \alpha_{1} \lambda_{1}^{2}, \\
\tilde{\Theta}_{2}= & \left(\gamma_{1}-\gamma_{2}\right)\left(\alpha_{1}-\beta_{1}\right), \\
\Theta_{3}= & \frac{\gamma_{1} \beta_{1} \lambda_{1}^{-2}-\gamma_{2} \beta_{1} \lambda_{1}^{2}+\gamma_{2} \alpha_{1} \lambda_{1}^{-2}-\gamma_{1} \alpha_{1} \lambda_{1}^{2}}{\lambda_{1}^{2}-\lambda_{1}^{-2}}, \\
\tilde{\Theta}_{3}= & 2\left(\gamma_{1}+\gamma_{2}\right)-\gamma_{1} \beta_{1} \lambda_{1}^{-2}-\gamma_{2} \beta_{1} \lambda_{1}^{2}-\gamma_{2} \alpha_{1} \lambda_{1}^{-2}-\gamma_{1} \alpha_{1} \lambda_{1}^{2}, \\
\Theta_{4}= & \left(\gamma_{1}-\gamma_{2}\right)\left(\alpha_{1}-\beta_{1}\right)+\left(\gamma_{1}+\gamma_{2}\right)\left(\lambda_{1}^{2}-\lambda_{1}^{-2}\right), \\
\tilde{\Theta}_{4}= & -\left(\lambda_{1}^{2}-\lambda_{1}^{-2}\right)\left(\gamma_{1}\left(\lambda_{1}^{-2} \beta_{1}+\lambda_{1}^{2} \alpha_{1}\right)+\gamma_{2}\left(\lambda_{1}^{2} \beta_{1}+\lambda_{1}^{-2} \alpha_{1}\right)\right) \\
& +\left(\lambda_{1}^{2}-\lambda_{1}^{-2}\right)\left(\left(\gamma_{1}+\gamma_{2}\right)\left(\alpha_{1}-1\right)\left(\beta_{1}-1\right)\right) \\
& +\gamma_{2}\left(\lambda_{1}^{2} \alpha_{1}-\lambda_{1}^{-2} \beta_{1}\right)+\gamma_{1}\left(\lambda_{1}^{2} \beta_{1}-\lambda_{1}^{-2} \alpha_{1}\right),
\end{aligned}
$$

and $\tilde{p}_{n}, \tilde{z}_{n}$ can be obtained directly from (32). Based on (32) and (34), we know that $\tilde{p}_{n}, \tilde{q}_{n}, \tilde{r}_{n}, \tilde{x}_{n}, \tilde{y}_{n}$ and $\tilde{z}_{n}$ are soliton solutions with free parameters $\lambda_{1}, \gamma_{1}, \gamma_{2}$. 


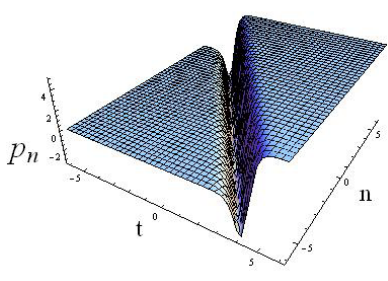

One-soliton solution $p_{n}$

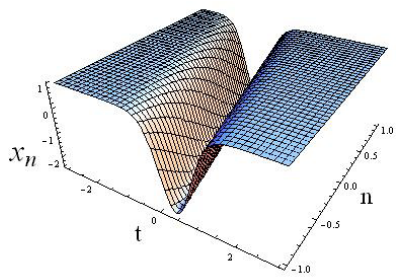

One-soliton solution $x_{n}$

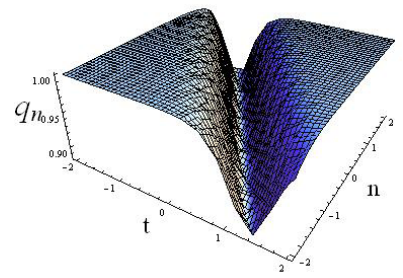

One-soliton solution $q_{n}$

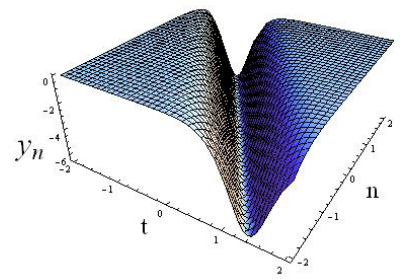

One-soliton solution $y_{n}$

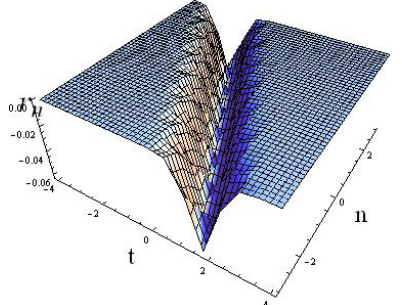

One-soliton solution $r_{n}$

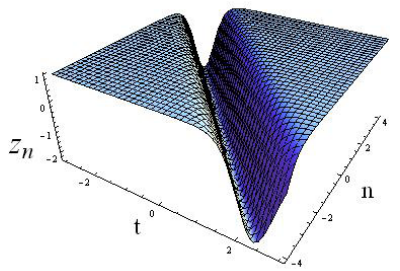

One-soliton solution $z_{n}$

Fig. 1. (Color online) Plot of the soliton solutions $p_{n}, q_{n}, r_{n}, x_{n}, y_{n}, z_{n}$ with the parameters $\lambda_{1}=0.5, \gamma_{1}=1$ and $\gamma_{2}=1.5$.

Next, we choose suitable parameters $\lambda_{i}$ and $\gamma_{i}$ to illustrate the structures of soliton solutions. When $\lambda_{1}=0.5, \gamma_{1}=1, \gamma_{2}=1.5$, Fig. 1 shows the bell-shaped one-soliton structure, respectively.

We remark that if the resulting solution is taken as the new starting point, and make the Darboux transformation once again, then another new solutions of (1) are obtained. This process can be done continuously. Therefore, we can obtain a sequence of explicit exact solutions for (1). We can also get the exact solution or soliton solutions of the self-dual network equation, the discrete second-order nonlinear Schrödinger equation and the relativistic Volterra lattice equation under the transformation (29) using the similar method.

Remark 4.1. We point out that by using the Darboux transformation in Refs. 12 and 14, Vakhnenko also obtained explicit analytical mulicomponent solutions with strictly defined free physical parameters: amplitude, velocity, energy, wideness of a soliton.

\section{Conclusion}

In this paper, we investigate a 6 -field integrable lattice system (1) under the spectral problem (5). Infinitely many conservation laws and a new Darboux transformation for (1) are derived by means of the Lax pair. As an application, explicit exact solutions are generated, from which we obtain the soliton solutions. The figures of the one-soliton solutions with properly parameters are presented to illustrate the 
soliton structures. In fact, with the help of Darboux transformation, the multisoliton solutions of system (1) can be obtained successfully.

\section{Acknowledgments}

This work was completed with the support by NSFC grant (Nos. 11771177, 11301210 and 11501242), China Automobile Industry Innovation and Development Joint Fund (No. U1664257), Program for Changbaishan Scholars of Jilin Province and Program for JLU Science, Technology Innovative Research Team (No. 2017TD-20), Science and Technology Development Project of Jilin Province (No. 20170520055JH) and the scientific research project of The Education Department of Jilin Province (No. JJKH20160398KJ).

We express our sincere thanks to the reviewers for the useful comments and hard work. Those comments are all valuable and helpful for revising and improving our paper as well as the important guiding significance to our researches.

\section{References}

1. E. Fermi et al., Studies of the nonlinear problems, Technical report, Los Alamos Scientific Lab. (New Mexico, 1955).

2. M. J. Ablowitz and J. F. Ladik, J. Math. Phys. 16, 598 (1975).

3. A. A. Belov and K. D. Chaltikian, Phys. Lett. B 309, 268 (1993).

4. M. Blaszak and K. Marciniak, J. Math. Phys. 35, 4661 (1994).

5. W. X. Ma and B. Fuchssteiner, J. Math. Phys. 40, 2400 (1999).

6. W. X. Ma and Y. C. You, Chaos, Solitons Fractals 22, 395 (2004).

7. W. X. Ma et al., Anal. Math. Phys. 8, 427 (2018).

8. W. X. Ma et al., Mod. Phys. Lett. B 32, 1850409 (2018).

9. W. X. Ma et al., J. Geom. Phys. 133, 10 (2018).

10. W. X. Ma et al., East Asian J. Appl. Math. 9, 100218 (2018).

11. W. X. Ma, Phys. Lett. A 379, 1975 (2015).

12. O. O. Vakhnenko, J. Math. Phys. 56, 033505 (2015).

13. O. O. Vakhnenko, J. Phys. A, Math. Gen. 39, 11013 (2006).

14. O. O. Vakhnenko, J. Phys. Soc. Jpn. 84, 014003 (2015).

15. T. Tsuchida, H. Ujino and M. Wadati, J. Math. Phys. 39, 4785 (1998).

16. R. Hirota, J. Phys. Soc. Jpn. 35, 289 (1973).

17. P. Yang, Y. Chen and Z. B. Li, Appl. Math. Comput. 210, 362 (2009).

18. X. Y. Wen, J. Phys. Soc. Jpn. 81, 114006 (2012).

19. X. Y. Wen, D. S. Wang and X. H. Meng, Rep. Math. Phys. 72, 349 (2013).

20. Y. B. Suris and O. Ragnisco, Commun. Math. Phys. 200, 445 (1999).

21. Z. N. Zhu, H. C. Huang and W. M. Xue, J. Phys. Soc. Jpn. 68, 771 (1999).

22. Z. N. Zhu et al., J. Phys. A, Math. Gen. 35, 5079 (2002).

23. K. Maruno and W. X. Ma, J. Nonlinear Math. Phys. 9, 127 (2002).

24. H. Q. Zhao, Appl. Math. Lett. 38, 79 (2014).

25. Y. T. Wu and X. G. Geng, J. Phys. A 31, L677 (1998).

26. X. X. Xu, Phys. Lett. A 362, 205 (2007).

27. E. G. Fan and H. H. Dai, Phys. Lett. A 372, 4578 (2008).

28. G. Z. Tu, J. Phys. A 23, 3903 (1990).

29. T. Tsuchida, H. Ujino and M. Wadati, J. Phys. A, Math. Gen. 32, 2239 (1999). 
30. D. J. Zhang and D. Y. Chen, Chaos, Solitons \& Fractals 14, 573 (2002).

31. O. O. Vakhnenko, J. Nonlinear Math. Phys. 18, 401 (2011).

32. O. O. Vakhnenko, J. Nonlinear Math. Phys. 24, 250 (2017).

33. H. W. A. Riaz et al., Commun. Nonlinear Sci. Numer. Simul. 48, 387 (2017).

34. X. J. Zhao, R. Guo and H. Q. Hao, Appl. Math. Lett. 75, 114 (2018).

35. N. Liu and X. Y. Wen, Mod. Phys. Lett. B 32, 1850085 (2018). 